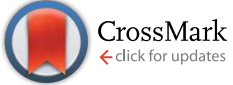

Cite this: RSC Adv., 2015, 5, 44865

\title{
Unconventional co-existence of plasmon and thermoelectric activity in In:ZnO nanowires $\dagger$
}

\author{
Alessandra Catellani, ${ }^{\text {ab }}$ Alice Ruini, ${ }^{\text {ac }}$ Marco Buongiorno Nardellide \\ and Arrigo Calzolari ${ }^{\star a d}$
}

Metal-doped $\mathrm{ZnO}$ nanowires exhibit the unique property of being simultaneously thermoelectric transparent conductors and low-loss plasmonic materials in the near-IR and visible range. Using calculations from first principles, we identify the mechanisms that regulate this behavior at the nanoscale and we describe how nanostructuring affects the optoelectronic, vibrational and transport properties of In: $\mathrm{ZnO}$ nanowires. Our results reveal that In doping imparts a good electrical conductivity and provides an injected free charge sufficient to sustain a surface-plasmon-polariton excitation. At the same time, surface scattering effects efficiently quench the thermal conductivity along the wire, improving the thermoelectric figure of merit of the system with respect to the bulk material. The coexistence of plasmonic and thermoelectric characteristics fosters the design of a novel class of coupled nanostructured devices for photothermal-electrical energy conversion.

Received 7th April 2015

Accepted 12th May 2015

DOI: $10.1039 / \mathrm{c} 5 \mathrm{ra06199g}$

www.rsc.org/advances

\section{Introduction}

Sunlight energy conversion throughout exciton dissociation in nanoscale systems (e.g. nanoparticles, nanowires, tetrapods, hierarchical structures, etc.) is a valuable alternative to carbonbased sources for green and low cost power generators. ${ }^{1}$ Yet, the shrinking of the solar cell dimensions causes relevant problems, especially for the local thermal dissipation that limits the efficiency of photovoltaic systems. ${ }^{2}$ On the contrary, thermophotovoltaic converters, ${ }^{3}$ which directly convert the energy of photons emitted by a thermal source into electrical energy, are limited to photons with energy above the bandgap, thus reducing the range of solar spectrum available for photoconversion.

The design of mixed architectures that could positively exploit both light and heat conversion would represent an important step forward in the realization of more efficient devices. Particularly promising are recent prototypical systems that couple a plasmonic heater to a thermoelectric device: ${ }^{4-6}$ the former acts as photothermal converter, through an excitationdissipation process mediated by a plasmon, ${ }^{7,8}$ which activates a

\footnotetext{
${ }^{a}$ Istituto Nanoscienze CNR-NANO-S3, I-41125 Modena, Italy. E-mail: arrigo. calzolari@nano.cnr.it; Fax: +39059 367488; Tel: +39059 2055627

${ }^{b}$ CNR-IMEM, Parco Area delle Scienze, 37A, I-43100 Parma, Italy

'Dipartimento di Fisica, Informatica e Matematica, Universitá di Modena e Reggio Emilia, I-41125 Modena, Italy

${ }^{d}$ Department of Physics, University of North Texas, Denton, TX 76203, USA

${ }^{e}$ Center for Materials Genomics, Duke University, Durham, NC 27708, USA

$\dagger$ Electronic supplementary information (ESI) available: ESI includes complementary details on the electronic structure, the real and imaginary part of the dielectric function and transport properties of $\mathrm{ZnO}$ and IZO wires. See DOI: $10.1039 / \mathrm{c} 5 \mathrm{ra} 06199 \mathrm{~g}$
}

thermoelectric power generator., ${ }^{9,10}$ Along these lines, Xiong and coworkers ${ }^{4}$ demonstrated that the power conversion efficiency of a commercial thermoelectric unit is enhanced by $\sim 5 \%$ upon coating with plasmonic Ag nanoparticles.

Despite these promising results, this technology is, however, in its very infancy and many challenges must be addressed to improve the efficiency of these devices. In particular, the choice of the materials is one of the most crucial problems, because in order to minimize losses and to maximize the thermal transfer between the plasmonic heater and the thermoelectric converter, the two systems should be prepared with the same material. ${ }^{11}$ This constitutes a strict limitation: in order to promote plasmonic excitations in the visible and near-IR range (i.e. the part of the spectrum that corresponds to the maximum of solar radiation) the plasmon heater must include metallic systems with a huge amount of free electrons. On the other hand, noble metals like silver or gold are not good thermoelectric materials, as they are very efficient thermal conductors. On the contrary, standard bulk thermoelectric materials are doped semiconductors with high electron mobility, and low thermal conductivity, but no plasmon activity in the low-energy region.

The search for materials that are simultaneously plasmonic and thermoelectric is a tremendous challenge. Among the potential candidates, ZnO seems to uniquely fulfill all the requirements. $\mathrm{ZnO}$ is a direct bandgap wurtzite semiconductor with high electrical conductivity, efficient luminescence and strong excitonic effects even at room temperature. Due to its large band gap $(3.4 \mathrm{eV})$, intrinsic $\mathrm{ZnO}$ is transparent and represents an attractive choice for applications in ultraviolet light emitters, field-effect transistors, sensors, piezoelectric devices and polariton laser. ${ }^{12}$ Upon metal doping (e.g. In, Al, 
$\mathrm{Ga}$ ), $\mathrm{ZnO}$ acts as a transparent conductive oxide (TCO $)^{\mathbf{1 3 - 1 5}}$ with a large amount of free conduction charge $\left(>10^{19} \mathrm{e} \mathrm{cm}^{-3}\right)$, able to support bulk plasmon oscillations and surface plasmon polaritons (SPP) in the visible and near-IR range, including the standard telecommunication wavelength $(1.5 \mu \mathrm{m}) .{ }^{16-18}$ Very recently, it has been also demonstrated that plasmonic $\mathrm{ZnO}$ nanowires can be used to realize coherent sources of surface plasmon polaritons at the nanoscale and surface plasmon emitting diodes. ${ }^{19}$

In virtue of the wurtzite structure, $\mathrm{ZnO}$ may be easily grown in ordered arrays of nanostructures oriented and elongated along the polar axis, ${ }^{20,21}$ such as wires and tetrapods, that have been largely applied in optoelectronic devices like solar cells and LEDs. ${ }^{22,23}$ Even though the intrinsic thermoelectric properties of bulk $\mathrm{ZnO}$ are rather poor, ${ }^{24} \mathrm{ZnO}$ nanowires have been recently used as active element in thermoelectric devices with enhanced $Z T$ figure of merit. ${ }^{25-27}$ In particular, two-terminal transport measurements on individual suspended $\mathrm{ZnO}$ nanowires in vacuum ${ }^{28}$ demonstrated that moderate doping can increase the electrical conductivity without decreasing the Seebeck coefficient and that thermal conductivity of ZnO NWs is much smaller than the bulk one over a very large range of temperature (300-1000 K).

Many fundamental issues, however, remain unclarified. For instance, there is no consensus on the possibility to highly dope nanostructures without compromising their structural and electronic stability ${ }^{\mathbf{2 9}, 30}$ or to induce a TCO behavior in 1D structures. Furthermore, it is not confirmed that metal doping may provide enough free conduction charge to the host to support plasmon excitations in nanowires. ${ }^{31}$ Finally, the effect of the boundary surfaces on the thermal transport of the wire and its effect on the thermoelectric properties is still an open question.

In this paper, we provide a first principles characterization of the electronic, plasmonic and thermoelectric properties of Indoped $\mathrm{ZnO}$ (IZO) nanowires, focusing on the effects of doping and of the presence of boundary surfaces on the intrinsic optoelectronic and transport properties of the wires. Our results confirm that arrays of IZO wires are suitable candidates to realize both plasmon heaters and thermoelectric generators, which can be integrated in a unique device, opening the way for a new generation of photothermal-electrical energy converters.

\section{Results and discussion}

\subsection{Optoelectronic and plasmonic properties}

We first characterize the structural and electronic properties of an undoped hexagonal $\mathrm{ZnO}$, taken as a reference. The simulated wire (Fig. 1) has a diameter of $1.7 \mathrm{~nm},{ }^{32}$ with the longitudinal axis parallel to the polar direction [0001] and exposes non-polar [10̄0] faces, as commonly observed in experiment. ${ }^{20}$ In the relaxed geometry, surface $\mathrm{Zn}-\mathrm{O}$ dimers buckle to minimize the effect of the surface dangling bonds. The ground state electronic Density Of States (DOS) is reported in Fig. 2a. Spectra over larger energy range, including semicore region, are shown in Fig. S1 of the ESI. $\dagger$ The use of the ACBN0 functional ${ }^{33}$ corrects almost completely the underestimation of the $\mathrm{ZnO}$ bandgap of

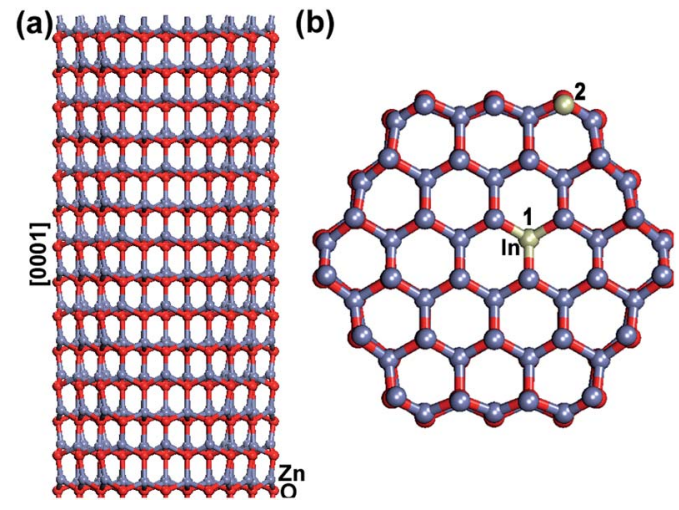

Fig. 1 Side (a) and top (b) view of In-doped $\mathrm{ZnO}$ wire. Labels 1 and 2 mark indium sites.

standard DFT, ${ }^{\mathbf{3 4}, 35}$ giving a Khon-Sham (KS) gap of $3.23 \mathrm{eV}$. The frontier orbitals have a $\pi$-like character with the highest occupied valence states localized around the external crown of the wire, and the lowest unoccupied conduction states localized in the central core of the wire, as shown in Fig. S2-S4 of ESI. $\dagger$ This is in agreement with previous theoretical calculations of similar ZnO wires, with different diameters. ${ }^{36,37}$

The experimental results ${ }^{38}$ show that within the solubility limit $(\sim 3.5 \%)$ group IIIA metals (Al, Ga, In) dope $\mathrm{ZnO}$ in $\mathrm{Zn}$ substitutional sites, with very similar effects. Indium, with its large ionic radius, is frequently used in lab experiments because it is easily recognizable through microscopy techniques. ${ }^{39}$ With respect to bulk case, when doping a nanowire we have to distinguish between external and internal defect sites. Here, we considered IZO wires obtained substituting one $\mathrm{Zn}$ atom from

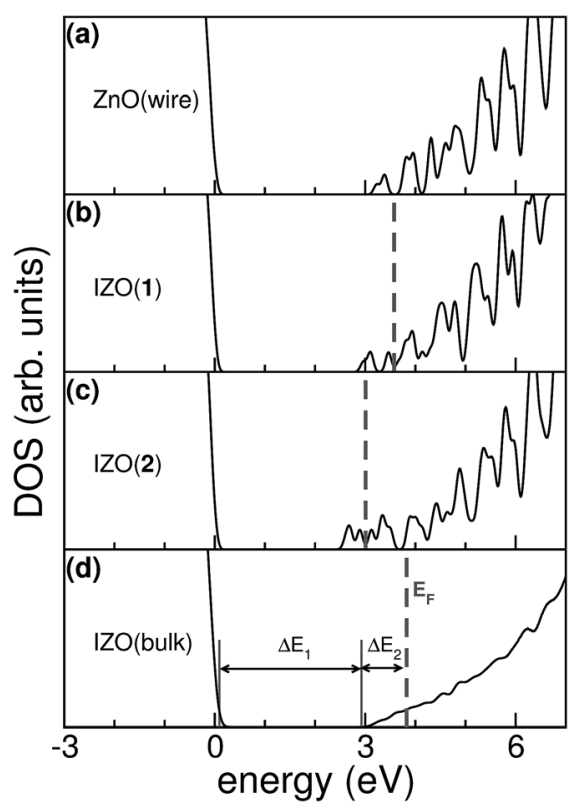

Fig. 2 DOS of (a) undoped ZnO wire, (b) IZO wire, configuration 1, (c) IZO wire, configuration 2, (d) IZO bulk. Dashed vertical lines identify the Fermi level of each metallic system. Zero energy reference is set to the valence band top of un-doped hosts. 
the center (position 1) or from the surface (position 2) of the wire, as shown in Fig. 1b. In both cases, the inclusion of the dopant does not perturb the crystalline order of the ZnO host, in agreement with what observed for the $3 \mathrm{D}$ bulk phases. This is a first fundamental result as the dopability of the nanostructures is a well-known challenge. ${ }^{\mathbf{4 0 , 4 1}}$ Due to the high surface-to-bulk ratio and the high reactivity of uncapped nanostructures, deriving from the abundance of frustrated bonds, the inclusion of dopants may indeed cause strong geometrical deformations that destabilize the structure and/or destroy the bond properties of the material under-/over-coordinating the atoms the host. This generally introduces trap states in the gap that are detrimental for the optical and transport properties of system. Thus, the structural stability of IZO nanowires is a fundamental prerequisite for the applications described above.

The DOS plots of IZO wires are displayed in panel (b) and (c) of Fig. 2, respectively; panel (d) shows the DOS for the 3D IZO bulk, in the wurtzite structure, included for comparison. From Fig. 2 it is evident that the DOS of the doped and undoped wires are qualitatively very similar to the $3 \mathrm{D}$ case, and the original bandgap of $\mathrm{ZnO}\left(\Delta E_{1}\right)$ is easily recognizable in all systems. However, while the presence of In in the $3 \mathrm{D}$ bulk does not change the shape and the curvature of the conduction band minimum, band modifications are more pronounced in $1 \mathrm{D}$ systems.

This is also reflected by the small but not-negligible differences induced by doping in the $\Delta E_{1}$ values (Table 1) for the wires, which accounts for a reduction of the internal ionicity, due to the presence of the In atoms. The inclusion of the dopant causes a decrease of the original $\mathrm{ZnO}$ gap $\left(\Delta E_{1}\right)$, which partially compensates the gap opening induced by quantum confinement. IZO wires further exhibit a flattening of the lowest conduction band, which corresponds to an increase of the electron effective masses $m_{\mathrm{e}}^{*}$ (Table 1 ). This effect is larger in the case of surface dopant (2), whose effective mass is almost twice the one of bulk-like defects (1).

For all IZO systems (wires and bulk), no defect states appear in the gap: indium donates its $5 \mathrm{p}$ electron to the $\mathrm{ZnO}$ host, shifting the Fermi level into the original conduction band of $\mathrm{ZnO}$. However, the amount of free electron charge injected in the $\mathrm{ZnO}$ matrix depends on the system. In order to quantify this effect, we defined the index $\Delta E_{2}$, which is the energy difference between the Fermi level of IZO and the conduction band minimum of ZnO, as shown in Fig. 2. The deeper the Fermi level

Table 1 Energy differences $\left(\Delta E_{1}\right.$ and $\left.\Delta E_{2}\right)$, effective mass $\left(m_{\mathrm{e}}^{*}\right)$, plasma frequency $\left(\omega_{\mathrm{p}}\right)$, free electron concentration $\left(n_{\mathrm{e}}\right)$ and optical gap $\left(E_{\mathrm{g}}^{\mathrm{opt}}\right)$ for undoped/doped nanowires and bulk. Bandstructure parameters $\Delta E_{1}$ and $\Delta E_{2}$ refer to Fig. 2

\begin{tabular}{lllllll}
\hline & $\Delta E_{1}(\mathrm{eV})$ & $\Delta E_{2}(\mathrm{eV})$ & $m_{\mathrm{e}}^{*} / m_{0}$ & $\omega_{\mathrm{p}}(\mathrm{eV})$ & $n_{\mathrm{e}}\left(\mathrm{cm}^{-3}\right)$ & $E_{\mathrm{g}}^{\mathrm{opt}}$ \\
\hline $\mathrm{ZnO}($ wire $)$ & 3.23 & - & 0.34 & - & - & 3.23 \\
$\mathrm{IZO}(1)$ & 2.94 & 0.67 & 0.38 & 0.64 & $1.1 \times 10^{20}$ & 3.85 \\
$\mathrm{IZO}(2)$ & 2.61 & 0.39 & 0.66 & 0.34 & $5.3 \times 10^{19}$ & 3.38 \\
ZnO(bulk) & 3.10 & - & 0.29 & - & - & 3.10 \\
IZO(bulk) & 3.13 & 0.80 & 0.29 & 1.24 & $3.1 \times 10^{20}$ & 4.07
\end{tabular}

is shifted into the conduction band (i.e. larger $\Delta E_{2}$ ), the larger is the charge injection. The best defect-to-host electron transfer is for IZO bulk (Table 1), followed by IZO(1) and IZO(2), whose $\Delta E_{2}$ is reduced by $\sim 50 \%$, with respect to the bulk case. We can thus conclude that In-doping imparts a n-type conductive character to $\mathrm{ZnO}$ wires, while the details of the electronic properties depend on the specific doping site: the inner site (1), thanks to the optimal coordination with the next-neighbor oxygen atoms, displays a very good charge delocalization, very similar to the $3 \mathrm{D}$ case. On the contrary, the under coordinated In-atom on the surface $(\operatorname{IZO}(2))$ favors a charge localization around the defect site and a reduction of the available free conduction charge. This is confirmed also by a residual magnetism for $\operatorname{IZO}(2)$ system, not detected in the other cases.

By using a semiclassical Drude-Lorentz model, based on independent particle band-to-band transitions, we calculated the complex dielectric function $\hat{\varepsilon}=\varepsilon_{1}+\mathrm{i} \varepsilon_{2}$ and the corresponding electron energy loss (EEL) function $L(\omega)=\operatorname{Im}\{-1 / \hat{\varepsilon}\}$. The spectral plots for the real and imaginary part of the dielectric function are shown in ESI (Fig. S5 $\dagger$ ). The results confirm that the undoped wire exhibits the typical features of a wide bandgap semiconductor. The inclusion of Indium imparts a metallic behavior in the infrared (i.e. negative $\varepsilon_{1}$ ), while it induces a blue-shift of the absorption edge in the UV-range, which preserves the transparency of the doped systems. The transparency in the visible range along with the electrical n-type characteristics confirms the TCO behavior displayed by both wire and bulk IZO compounds. The demonstration of TCO-like properties for the wire systems is a particularly important result, as low-dimensional TCO materials may be exploited as transparent contacts in a large range of optoelectronic nanostructured devices.

Fig. 3 shows the EEL spectra for the IZO materials. When $\varepsilon_{1}=0$ and $\varepsilon_{2} \ll 1$, the loss function has a peak, corresponding to the plasma frequency $\omega_{\mathrm{p}}$ of the system (Table 1 ). As the plasma frequency lies in the near-IR for both wires and bulk, while the absorption energy is the UV, we expect a drastic reduction of the energy losses due to interband transitions in the NIR-vis operating range, the one with the maximum sunlight power distribution. This would optimize the conversion of the incoming radiation into thermal energy through the excitation/deexcitation of plasmon resonances, and minimize the energy dissipation via absorption/emission radiative processes.

In agreement with the analysis of the electronic structure, we identify some numerical differences in the energy position $\omega_{\mathrm{p}}$, despite the fact that three systems have the same formal doping level. This can be directly related to the amount of injected free electron charge. Starting from the definition of the plasma frequency $\omega_{\mathrm{p}}=\sqrt{\frac{e^{2} n_{\mathrm{e}}}{\varepsilon_{0} m_{\mathrm{e}}^{*}}}$ (where $e$ is the electron charge, $\varepsilon_{0}$ the dielectric permittivity of vacuum and $m_{\mathrm{e}}^{*}$ the electron effective mass), we extracted the free electron density $n_{\mathrm{e}}$. Consistently with the DOS results presented above, configuration (2) presents lower available charge than configuration (1) and IZO bulk. The resulting values for the IZO wires are however larger than 


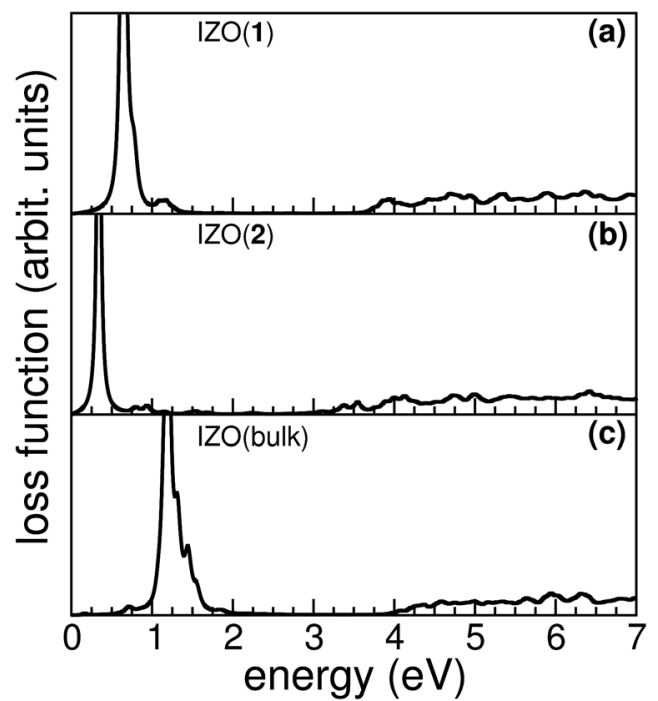

Fig. 3 EELS spectra of (a) undoped $\mathrm{ZnO}$ wire, (b) IZO wire, configuration 1, (c) IZO wire, configuration 2, (d) IZO bulk.

the empirical lower concentration $\left(10^{19} \mathrm{~cm}^{-3}\right)$, required to sustain a plasma excitation.

The interface between IZO wires and vacuum can be considered as the simplest metal/dielectric interface, along which it is possible to excite a surface-plasmon polariton. As in our simulation we explicitly take into account the effect of the surface/vacuum interface, the calculated $\omega_{\mathrm{p}}$ can be directly assumed as the frequency of the surface plasmon polariton $\omega_{\text {spp }}$. Notably, the ideal SPP frequency obtained from the expression $\omega_{\text {spp }}=\omega_{\mathrm{p}}^{\text {bulk }} / \sqrt{2}=0.88 \mathrm{eV}$ is only slightly larger than the value directly obtained from IZO wire (Table 1). This difference accounts for the confinement effect acting on the true 1D systems and absent in bulk calculations.

We can conclude that In-doped $\mathrm{ZnO}$ nanowires act as $1 \mathrm{D}$ TCO materials that can be exploited as low loss plasmonic elements in the near-IR and visible range. Thus, they are very promising candidates to realize a plasmon heater, i.e. converting the solar light into thermal radiation through the excitation/ de-excitation of a SPP along the wire.

\subsection{Transport properties}

For ultrasmall nanowires, as the ones we are considering here, the scattering contributions with the boundaries, due to extremely high surface-to-volume ratio, are the predominant effects that affect both electron and thermal transport. ${ }^{42,43}$ Thus, the coherent regime is a good first approximation for the description of transport in a large range of temperature, almost up to room temperature. For larger diameter wires or for very high temperature, the transport becomes rather diffusive and dissipative scattering terms (e.g. el-el, el-ph, ph-ph) should be carefully included.

We simulated both electron and thermal coherent transport along $\mathrm{ZnO}$ nanowires by using the WanT code,${ }^{44}$ which exploits the complete energy band structures and phonon dispersions to solve an extended Landauer problem, within a real-space
Green's function framework. This approach allows us to directly link the transport properties (e.g. quantum conductance and $I / V$ characteristics) to the dimensionality, and atomistic structure of the system. The method holds for a generic two-terminal open device (left-lead/conductor/right-lead). We here focus on the intrinsic conduction properties of the wires that are the theoretical prerequisite for any further two-terminal device simulation. This is easily done considering the two external leads made of the same material of the conductor (i.e. the $\mathrm{ZnO}$ wire). We refer to original papers ${ }^{45-48}$ for the complete description of the theory and the code implementation.

In the Landauer approach, a key quantity to be calculated is the quantum transmittance, which represents the probability that a carrier (either electrons or phonons) may cross the conductor at a certain energy. The electron $\left(\mathscr{T}_{\text {el }}\right)$ and phonon $\left(\mathscr{T}_{\mathrm{ph}}\right)$ transmittance plots are shown in panels $(\mathrm{a}-\mathrm{c})$ and $(\mathrm{d})$ of Fig. 4, respectively (see ESI $\dagger$ for further details). The electronic contributions have been explicitly evaluated for the $\mathrm{ZnO}$ and the two IZO wires under investigation. In the case of thermal transport, as the In-substitution only slightly perturbs the atomic structure, we calculated the $\mathscr{T}_{\text {ph }}$ function only for the undoped $\mathrm{ZnO}$ wire, assuming as negligible the effect of Indium. In order to prove this statement, we checked a posteriori that the vibrational properties and, thus, the thermal transport were not changed, when the inclusion of In was considered in the mass defect approximation. ${ }^{49}$

All the spectra in Fig. 4 have a step-like behavior typical of periodic systems. In the absence of external leads, at a given value of energy (wavenumber), the quantum transmittance is constant and proportional to the number of transmitting channels available for charge (phonon) mobility, which are equal to the number of conducting bands at the same energy
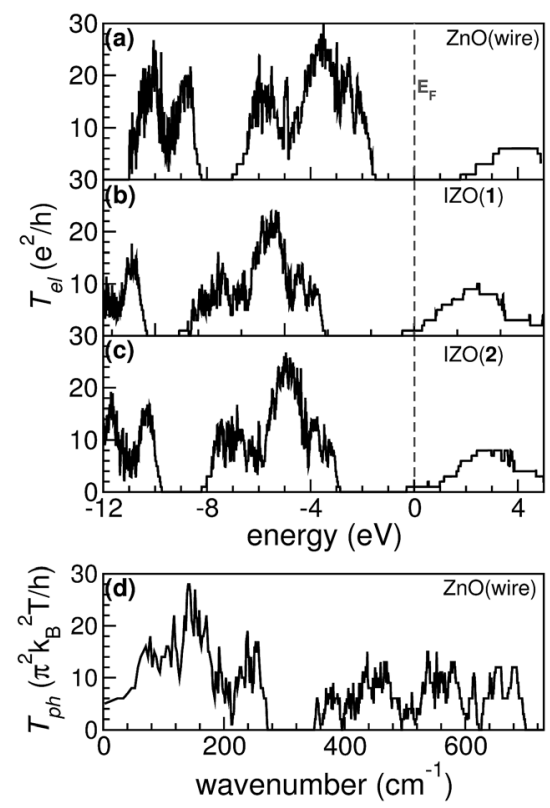

Fig. 4 Electron transmittance for (a) undoped $\mathrm{ZnO}$ wire, (b) IZO wire, configuration 1, (c) IZO wire, configuration 2. (d) Phonon thermal transmittance for un-doped $\mathrm{ZnO}$ wire. 
(see also Fig. S6, ESI $\dagger$ ). The electron quantum transmittance in particular well describes the electron donor effect due to doping, along with the quantitative differences in the position of the Fermi level between internal and external doping sites.

Although in periodic systems there is a direct correlation between transmittance and band structure, not all the states contribute to transport. This is particularly important in the case of thermal transport, that should be as low as possible in order to maximize the thermoelectric response of the system. In order to understand the features of the thermal conductance, we have carried out a microscopic analysis of the transmittance in terms of the most relevant contribution from the vibrational normal modes. The phonon DOS of the $\mathrm{ZnO}$ wire is shown in Fig. 5a, along with the corresponding bulk one, taken as reference. Following the irreducible representation of the wurtzite symmetry group $C_{6 \mathrm{v}}$, the phonon modes of $\mathrm{ZnO}$ bulk can be classified as $\Gamma=2 A_{1}+2 B_{1}+2 E_{1}+2 E_{2}$. One low energy $A_{1}$ and one double $E_{1}$ modes correspond to the transverse and longitudinal acoustic branches, while the others are optical modes.

In the nanowire, the presence of the surface breaks the atom equivalence of the bulk, introducing new phonon modes. This results in the spread of optical phonon branches at about $\sim 350-430$ and at $600-700 \mathrm{~cm}^{-1}$ and in a general redistribution of the spectral weight in the entire wavenumber range, in agreement with Raman experimental data. ${ }^{\mathbf{5 0}}$ The rotational invariance along the wire axis gives rise to a fourth zero-frequency mode at $\mathbf{q}=0$. This can be directly detected in the transmittance, which is $\mathscr{T}_{\mathrm{ph}}=4$, in the zerofrequency limit. From the analysis of the single phonon displacements we can also distinguish between core-longitudinal and surf-transverse modes. The former contribute to
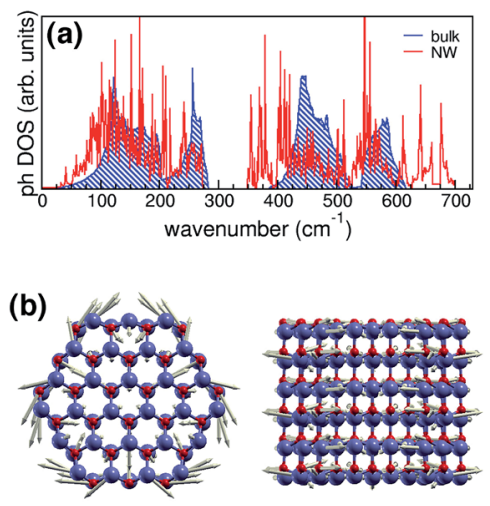

(c)
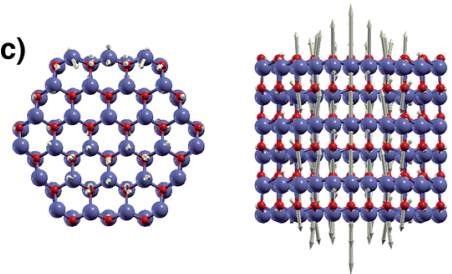

Fig. 5 (a) Phonon density of states of ZnO bulk (shaded area) and wire (thick line). Top (left) and side (right) view of atomic displacement of selected phonon modes at (b) $99.6 \mathrm{~cm}^{-1}$ (surf-transverse mode) and (c) $88.2 \mathrm{~cm}^{-1}$ (core-longitudinal mode). coherent thermal transport, the latter do not transmit along the wire. Two representative phonon modes are displayed in panels (b and c) of Fig. 5. It is evident that the surface acts as an extended scattering defect that blocks part of the phonon modes. This is particularly true for ultrathin nanowires, where the ratio between surface and core modes is very high. The phonon component of the thermal conductance $\left(K_{\mathrm{ph}}\right)$, resulting from the integration of the corresponding thermal transmittance $\mathscr{T}_{\mathrm{ph}}$ (see Fig. S6, ESI $\dagger$ ) at $T=300 \mathrm{~K}$ is $\sim 2 \mathrm{nW}$ $\mathrm{K}^{-1}$, i.e. one order of magnitude less than the corresponding bulk value. This goes in the direction of intrinsic reduction of the phonon thermal transport, as desired for thermoelectric application.

In the coherent transport regime, the figure of merit reduces to $Z T=S^{2} G_{\mathrm{el}} T / K_{\mathrm{t}}$, where $G_{\mathrm{el}}, K_{\mathrm{t}}$ are the electron and thermal conductances, respectively (see ESI $\dagger$ ). Once the thermal transmittance functions $\mathscr{T}_{\text {el }}$ and $\mathscr{T}_{\text {ph }}$ are known, the thermopower coefficient and the thermoelectric $Z T$ figure of merit can be straightforwardly obtained by using standard kinetic relations $^{51}$ (see ESI $\dagger$ ). The calculated $Z T$ plots for the undoped and doped wires are displayed in Fig. 6 in the temperature range 200-400 K. The figure of merit generally increases with temperature, while the dependence on the chemical potential depends on the original position of the Fermi level of the system. For instance, the edge position of the $\mathrm{ZnO}$ wire at $\sim 1.75 \mathrm{eV}$ reflects the presence of an energy gap. The absolute value of $Z T$ is instead directly related to the intrinsic electron conductance (e.g. effective mass) of the system, so the $Z T$ value of the undoped wire is almost double the value of IZO wires. The calculated $Z T$ values for the wire are about one order of magnitude larger than the corresponding measured value for the $\mathrm{ZnO}$ bulk $(Z T<0.001)$ and are in very good agreement with the experimental results for $\mathrm{Al}$-doped $\mathrm{ZnO}$ wires at the same temperatures. ${ }^{52}$ This confirms that: (i) the coherent transport approximation is accurate enough to describe the thermoelectric properties of ultrathin nanowires and (ii) nanostructuring is a promising way to increase the thermoelectric response of materials.

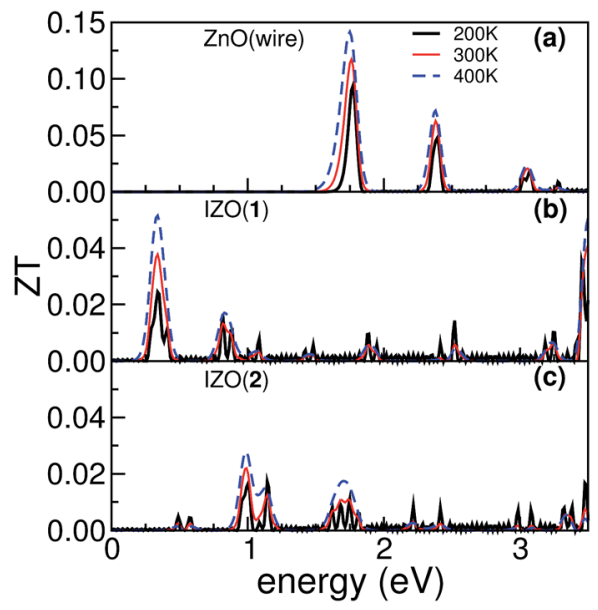

Fig. 6 (a) $Z T$ figure of merit evaluated at $T=300 \mathrm{~K}$ for (a) undoped $\mathrm{ZnO}$ wire, (b) IZO wire, configuration 1, (c) IZO wire, configuration 2. 


\section{Conclusions}

Using calculations from first principles, we have characterized In-doped $\mathrm{ZnO}$ nanowires and derived a number of relevant properties that are crucial for a better control of these plasmonic and thermoelectric nanostructures: (i) notwithstanding the high surface-to-volume ratio and reactivity of the system, intentional doping up to the solubility limit does not perturb the stability and crystalline structure of the wire, and imparts similar electronic properties as in the bulk case. This causes the Drude-like description of the plasmonic properties, which derive from the free residual charge in the conduction band of the nanowires; (ii) the doped wire itself behaves as a TCO, thus opening the possibility of designing TC nanodevices; (iii) the calculated thermoelectric figure of merit $Z T$ of the nanowires is almost twice as large as the one of the bulk, confirming that nanostructuring can provide a route to optimize the thermoelectric power.

This unusual coexistence of plasmonic and thermoelectric properties may be exploited to realize coupled surface-plasmon/ thermoelectric power generators where both active elements are realized with arrays of IZO wires. An illustrative scheme of this kind of device is displayed in Fig. 7. The nano-heater converts the absorbed photons into heat (i.e. photothermal effect) through the excitation of a surface plasmon polariton (SPP) along the wire. The photothermal effect involves complex excitation-dissipation processes that include: ${ }^{7,8}$ the excitation of plasma oscillations in a quasi-free electron gas; the ultrafast thermalization of the hot-electron gas through inelastic electron-electron scattering; the energy transfer to crystal lattice via phonon emission (electron-phonon coupling) ${ }^{53}$ and thermal radiation (heat diffusion) between nanowires and towards the external environment. The thermal energy resulting from the plasmon decay can be exploited to generate a temperature gradient across the thermoelectric unit, whose temperature is controlled by the presence of thermal bath. An intermediate gap spacer between the two units of the device prevents the overheating of the thermoelectric part, which is detrimental for the energy conversion efficiency. The manufacturing of such a

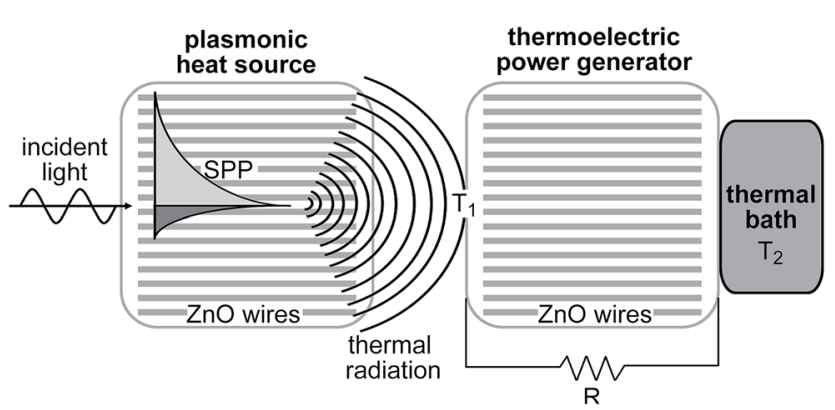

Fig. 7 Schematic model of a coupled surface-plasmon/thermoelectric power generator based on ZnO nanowires. An incident light excites a surface plasmon polariton (SPP) in the heat source part. The SPP thermal relaxation generates a temperature gradient $\left(T_{1} \neq T_{2}\right)$ in the thermoelectric part, giving rise to power available for an external resistance load $(R)$ device would constitute a major step forward in the quest for efficient systems for green energy conversion.

\section{Methods}

Ground state structural and electronic structure optimization is carried out by using total-energy-and-forces approaches, based on Density Functional Theory (DFT), as implemented in the QUANTUM ESPRESSO suite of codes. ${ }^{54}$ Single particle wavefuctions (charge) are expanded in a planewave basis set, up to an energy cutoff of 28 Ry (280 Ry). Atomic potentials for each chemical specie are described by Vanderbilt like ultrasoft psudopotentials: ${ }^{55} \mathrm{Zn}$ 3d and In 4d electrons are explicitly included in the valence shell.

The pathological underestimation of the ZnO bandgap (characteristic of the standard DFT semi-local approaches) is corrected by using the newly developed ACBNo functional. ${ }^{33}$ ACBN0 is a pseudo-hybrid Hubbard density functional that is a fast, accurate and parameter-free extension of traditional DFT + $U$ that has been proved to correct both the band gap and the relative position of the different bands in transition metal compounds, in particular the ones deriving from the d orbitals of transition metal atoms. Within ACBN0, the values of $U$ and $J$ are functionals of the electron density and depend directly on the chemical environment and crystalline field, thus providing a direct way of computing the Hubbard corrections for any individual atom in any local environment. ACBNO selfconsistent $\mathrm{U}$ values are $12.8 \mathrm{eV} 5.29 \mathrm{eV}$ for the $3 \mathrm{~d}$ orbitals of zinc and $2 p$ orbitals of oxygen, respectively, in perfect agreement with previous parameters reported by Calzolari et al. ${ }^{34}\left(\mathrm{U}_{3 \mathrm{~d}}\right.$ $\left.=12.0, \mathrm{U}_{2 \mathrm{p}}=6.5 \mathrm{eV}\right)$ and Ma et al. ${ }^{56}\left(\mathrm{U}_{3 \mathrm{~d}}=10, \mathrm{U}_{2 \mathrm{p}}=7 \mathrm{eV}\right)$, both of which were found by a fitting procedure to reproduce the experimental bandgap and position of the $3 \mathrm{~d}$ bands.

ZnO nanowires are simulated by using periodically repeated orthorhombic supercells (108 atoms). Parallel replica are separated by $\sim 12 \AA$ of vacuum in the directions perpendicular to the wire axis. IZO wires are obtained substituting one $\mathrm{Zn}$ atom with an In one in different positions (see Fig. 1), this corresponds to formal doping of $\sim 1.0 \% .10 k$-points along the wire axis are used to sample the 1D Brillouin Zone (BZ). 3D IZO bulk is simulated by using a hexagonal $(3 \times 3 \times 3) \mathrm{ZnO}$ supercell, also including 108 atoms; i.e. corresponding to the same doping value. In this case, a $(6 \times 6 \times 6) k$-point mesh is used for the $\mathrm{BZ}$ sampling. All structures were relaxed until forces on all atoms were lower than $0.03 \mathrm{eV} \AA^{-1}$.

The complex dielectric function $\hat{\varepsilon}$ is calculated in the independent particle approximation, using the code epsilon.x, also included in the QUANTUM ESPRESSO suite. The code implements a band-to-band formulation of the Drude-Lorentz model for solids. ${ }^{57,58}$ The complete theoretical treatment and the accuracy tests for the case of Al-doped $\mathrm{ZnO}$ system can be found in ref. 18. In this case DFT calculations are performed employing norm conserving pseudopotentials with an energy cutoff of $100 \mathrm{Ry}$. Once the complete dielectric function is known, the electron energy loss function can be easily obtained as $L(\omega)=\operatorname{Im}\{-1 / \hat{\varepsilon}\}$. 
Electronic and thermal coherent transport characteristics are simulated by using the WanT package, ${ }^{44}$ which provides a unified real-space implementation of the Landauer theory based on Green's function technique, ${ }^{45,59}$ for both electrons ${ }^{46}$ and phonons. ${ }^{48}$ The real space electronic hamiltonian is obtained from the DFT calculation through a pseudo-atomic projection procedure, as described in ref. 47. The phonon spectrum and Interatomic Force Constant (IFC) matrix are simulated with a joint finite-differences/finite-fields approach, also implemented in the QUANTUM ESPRESSO/package. ${ }^{60}$ For the calculation of phonon modes we considered a $(3 \times 1 \times 1)$ orthorhombic supercell (324 atoms) and we calculated forces (i.e. IFC) displacing only 108 atoms in primitive cell along the three spatial directions. This corresponds to a set of 324 DFT calculations.

\section{Acknowledgements}

MBN acknowledges partial support from ONR-MURI under contract N00014-13-1-0635. AC acknowledges funding from the European Union Seventh Framework Programme under Grant Agreement ITN-Nanowiring (Grant no. 265073). CINECA Computing Center (ISCRA Project IsB07_TherZnO) and Texas Advanced Computing Center (TACC) at the University of Texas Austin are acknowledged for providing HPC resources.

\section{References}

1 T. Soga, Nanostructured Materials for Solar Energy Conversion, Academic Press Elsevier, Amsterdam, NL, 2006.

2 A. Royne, C. J. Dey and D. R. Mills, Sol. Energy Mater. Sol. Cells, 2005, 86, 451-483.

3 T. Bauer, Thermophotovoltaics. Basic Principles and Critical Aspects of System Design, Springer, Berlin, Heidelberg, GE, 2011.

4 Y. Xiong, R. Long, D. Liu, X. Zhong, C. Wang, Z.-Y. Li and Y. Xie, Nanoscale, 2012, 4, 4416-4420.

5 J.-C. Weeber, K. H. Bouhelier, G. C. des Francs, J. Arocas, L. Markey and A. Dereux, Appl. Phys. Lett., 2011, 99, 031113 (1-3).

6 T. Hosseini and N. A. Kouklin, Appl. Phys. Lett., 2014, 105, 043104 (1-3).

7 M. Aeschlimann, Electron Dynamics in Metallic Nanoparticles, in Encyclopedia of Nanoscience and Nanotechnology, ed. N. Nalwa, American Scientific Publishers, USA, 2004.

8 R. Rosei, F. Antongeli and U. M. Grassano, Surf. Sci., 1973, 37, 689-699.

9 A. F. Bagley, S. Hill, G. S. Rogers and S. N. Bhatia, ACS Nano, 2013, 7, 8089-8097.

10 A. O. Govorov, W. Zhang, T. Skeini, H. Richardson, J. Lee and N. A. Kotov, Nanoscale Res. Lett., 2006, 1, 84-90.

11 R. Young, A. Narayanaswamy and G. Chen, J. Comput. Theor. Nanosci., 2013, 7, 8089-8097.

12 N. H. Nickel and E. Terukov, Zinc Oxide - A Material for Microand Optoelectronic Applications, NATO Science Series, Springer, AA Dordrecht, The Netherlands, 2006.
13 K. Ellmer, Nat. Photonics, 2012, 6, 809-817.

14 M. Bazzani, A. Neroni, A. Calzolari and A. Catellani, Appl. Phys. Lett., 2011, 98, 121907 (1-3).

15 M. Abb, B. Sepulveda, H. M. H. Chong and O. L. Muskens, J. Opt., 2012, 14, 114007 (1-7).

16 M. A. Noginov, L. Gu, J. Livenere, G. Zhu, A. K. Pradhan, R. Mundle, M. Bahoura, Y. A. Barnakov and V. A. Podolskiy, Appl. Phys. Lett., 2011, 99, 021101 (1-3).

17 G. V. Naik, V. M. Shalaev and A. Boltasseva, Adv. Mater., 2013, 25, 3264-3294.

18 A. Calzolari, A. Ruini and A. Catellani, ACS Photonics, 2014, 1, 703-709.

19 S. Wuestner, J. M. Hamm, A. Pusch and O. Hess, Laser Photonics Rev., 2015, 9, 256-262.

20 M. Law, L. E. Greene, J. C. Johnson, R. Saykally and P. Yang, Nat. Mater., 2005, 4, 455-459.

21 S. Singamanemi, M. Gupta, R. Yang, M. M. Tomczak, R. R. Naik, Z. L. Wang and V. V. Tsukruk, ACS Nano, 2009, 3, 2593-2600.

22 Y. W. Heo, D. P. Norton, L. C. Tien, Y. Known, B. S. Kang, F. Ren, S. J. Pearton and J. R. LaRoche, Mater. Sci. Eng., R, 2004, 47, 1-47.

23 Z. L. Wang, Mater. Sci. Eng., 2009, 64, 33-71.

24 T. Tsubota, M. Ohtaki, K. Eguchi and H. Arai, J. Mater. Chem., 1997, 7, 85-90.

25 L. Brockway, V. Vasiraju, M. K. Sunkara and S. Vaddiraju, ACS Appl. Mater. Interfaces, 2014, 6, 14923-14930.

26 A. J. Kulkarni and M. Zhou, Nanotechnology, 2007, 18, 435706 $(1-6)$.

27 C.-H. Lee, G.-C. Yi, Y. M. Zuev and P. Kim, Appl. Phys. Lett., 2009, 94, 022106 (1-3).

28 Y. Liu, Z. Zhang, X. Wei, Q. Li and L.-M. Peng, Adv. Funct. Mater., 2011, 21, 3900-3906.

29 S. T. Rajan, B. Subramanian, A. K. Kumar, M. Jayachandran and M. S. Ramachandra Rao, J. Alloys Compd., 2014, 584, 611-616.

30 C.-Y. Wang, C.-L. Kuo, C.-P. Liu, T.-Y. Wang, R.-K. Zheng and S. P. Ringer, J. Nanosci. Nanotechnol., 2011, 11, 10182 (1-6).

31 X.-Y. Zhang, A. Hu, T. Zhang, X.-J. Xue, J. Z. Wen and W. W. Duley, Appl. Phys. Lett., 2010, 96, 043109 (1-3).

32 Despite the ultrasmall size of the simulated NWs, their band-like electronic properties are still representative of the experimental ones (see ref. 23). Other properties (e.g. exciton binding energy, optical gap) that are more sensitive to the geometrical size and quantum confinement are, instead, less relevant in the spirit of the present work.

33 L. A. Agapito, S. Curtarolo and M. Buongiorno, Phys. Rev. X, 2015, 5, 011006 (1-16).

34 A. Calzolari, A. Ruini and A. Catellani, J. Am. Chem. Soc., 2011, 133, 5893-5899.

35 The ACBN0 method does not include many-body exciton's effects. As the plasmonic excitations (near-IR) are very well separated in energy from the excitonic peak (UV), the absence of excitonic contributions is irrelevant for the present purposes.

36 X. Q. Meng, H. Peng, Y. Q. Gai and J. Li, J. Phys. Chem. C, 2010, 114, 1467-1471. 
37 J. Schrier, D. O. Demchenko, L.-W. Wang and A. P. Alivisatos, Nano Lett., 2007, 7, 2377-2382.

38 H. Mondragón-Suárez, A. Maldonado, M. de la L Olvera, A. Reyes, R. Castanedo-Pérez, G. Torres-Delgado and R. Asomoza, Appl. Surf. Sci., 2002, 193, 52-59.

39 Y. W. Chen, Y. C. Liu, S. X. Lu, C. S. Xu, C. L. Shao, C. Wang, Y. Y. Zhang, Y. M. Lu, D. Z. Shen and X. W. Fan, J. Chem. Phys., 2013, 123, 134701 (1-5).

40 J. R. Chelikowsky, M. M. G. Alemany, T.-L. Chan and G. M. Dalpian, Rep. Prog. Phys., 2011, 74, 046501 (1-29).

41 D. Calestani, S. C. Dhanabalan, M. Villani, L. Lazzarini and A. Zappettini, CrystEngComm, 2014, 16, 1696-1701.

42 E. B. Ramayya, L. N. Mauer, A. H. Davoody and I. Knezevic, Phys. Rev. B: Condens. Matter Mater. Phys., 2012, 86, 115328 (1-11).

43 N. Mingo and L. Yang, Phys. Rev. B: Condens. Matter Mater. Phys., 2003, 68, 245406 (1-12).

44 WANT code by A. Ferretti, L. Agapito, A. Calzolari and M. Buongiorno Nardelli, http://www.wannier-transport.org.

45 M. Buongiorno Nardelli, Phys. Rev. B: Condens. Matter Mater. Phys., 1999, 60, 7828-7833.

46 A. Calzolari, N. Marzari, I. Souza and M. Buongiorno Nardelli, Phys. Rev. B: Condens. Matter Mater. Phys., 2004, 69, 035108 (1-10).

47 L. A. Agapito, A. Ferretti, A. Calzolari, S. Curtarolo and M. Buongiorno Nardelli, Phys. Rev. B: Condens. Matter Mater. Phys., 2013, 88, 165127(1-7).

48 A. Calzolari, T. Jayasekera, K. Kim and M. Buongiorno Nardelli, J. Phys.: Condens. Matter, 2012, 492204, 492204 (1-10).

49 S. Baroni, P. Giannozzi and E. Molinari, Phys. Rev. B: Condens. Matter Mater. Phys., 1990, 41, 3870(R).

50 X.-W. Fu, Z.-M. Liao, R. Liu, J. Xu and D. Yu, ACS Nano, 2013, 7, 1-20.

51 G. Grosso and G. Pastori Parravicini, Solid State Physics, Academic Press Elsevier Ltd., Amsterdam NL, 2nd edn, 2014.
52 X. Qua, W. Wang, S. Lv and D. Jia, Solid State Commun., 2011, 151, 332-336.

53 The fact that for moderate temperatures the inelastic scattering terms are not dominant in ultrasmall nanowires transport is not in contradiction with the role that such diffusive terms play in the plasmon relaxation process (photothermal effect): in the heater part the electron and phonon distributions are highly out-of-equilibrium and the local temperature may be locally very high, favoring the thermalization via energy dissipation. Different is instead the case for the thermoelectric part, whose temperature is controlled by the presence of thermal bath connect to the system.

54 P. Giannozzi, S. Baroni, N. Bonini, M. Calandra, R. Car, C. Cavazzoni, D. Ceresoli, G. L. Chiarotti, M. Cococcioni, I. Dabo, A. Dal Corso, S. de Gironcoli, S. Fabris, G. Fratesi, R. Gebauer, U. Gerstmann, C. Gougoussis, A. Kokalj, M. Lazzeri, L. Martin-Samos, N. Marzari, F. Mauri, R. Mazzarello, S. Paolini, A. Pasquarello, L. Paulatto, C. Sbraccia, S. Scandolo, G. Sclauzero, A. P. Seitsonen, A. Smogunov, P. Umari and R. M. Wentzcovitch, J. Phys.: Condens. Matter, 2009, 21, 395502, See also: http:// www.quantum-espresso.org.

55 D. Vanderbilt, Phys. Rev. B: Condens. Matter Mater. Phys., 1990, 41, R7892-R7895.

56 X. Ma, Y. Wu, Y. Lv and Y. Zhu, J. Phys. Chem. C, 2013, 117, 26029-26039.

57 F. Wooten, Optical Properties of Solids, Academic Press, Inc., San Diego, CA, 9211 USA, 1972.

58 R. Colle, P. Parruccini, A. Benassi and C. Cavazzoni, J. Phys. Chem. B, 2007, 111, 2800-2805.

59 S. Datta, Electronic transport in mesoscopic systems, Cambridge University Press, Cambridge, UK, 1995.

60 A. Calzolari and M. Buongiorno Nardelli, Sci. Rep., 2013, 3, 2999 (1-6). 